\title{
Análisis numérico tridimensional de la convección forzada para un flujo laminar sobre un escalón al frente
}

\section{Tri-Dimensional Numerical Analysis for Forced Convection over a Forward Facing Step}

\author{
J.G. Barbosa-Saldaña \\ SEPI-ESIME Unidad Profesional Adolfo López Mateos, \\ Instituto Politécnico Nacional. México. \\ LABINTHAP \\ E-mail:jbarbosas@ipn.mx \\ J.A. Jiménez-Bernal \\ SEPI-ESIME Unidad Profesional Adolfo López Mateos, \\ Instituto Politécnico Nacional. México. \\ LABINTHAP \\ E-mail:jjimenezb@ipn.mx \\ C. del C. Gutiérrez-Torres \\ SEPI-ESIME Unidad Profesional Adolfo López Mateos, \\ Instituto Politécnico Nacional. México. \\ LABINTHAP \\ E-mail:cgutierrezt@ipn.mx \\ P. Quinto-Diez \\ SEPI-ESIME Unidad Profesional Adolfo López Mateos, \\ Instituto Politécnico Nacional. México. \\ LABINTHAP \\ E-mail:pdq510@hotmail.com
}

(Recibido: octubre de 2007; aceptado: enero de 2009)

\section{Resumen}

Se desarrolla un código numérico basado en la técnica de los volúmenes finitos para simular las estructuras de flujo y la transferencia de calor en un ducto horizontal que encierra un escalón. El campo de velocidades y la distribución del campo de presión se relacionan numéricamente por medio del algoritmo SIMPLE. Las dimensiones del ducto se fijan en función de la altura del escalón, de tal forma que la relación de aspecto y la relación de expansión son igual a cuatro y dos, respectivamente. La longitud del ducto en la dirección principal del flujo es de sesenta veces la altura del escalón, mientras que éste se coloca a una distancia de veinte veces la altura del mismo, a partir de la entrada. Las condiciones de flujo a la entrada corresponden a las de un flujo completamente desarrollado a una temperatura constante $T_{0}$. Las condiciones de calentamiento se establecen considerando que la pared inferior está a una temperatura constante $T_{w}$, de tal forma que $T_{w}>T_{0}$, mientras que las paredes restantes se consideran adiabáticas. En este trabajo se presentan resultados de la posición y tamaño de las zonas de recirculación, así como de las estructuras de flujo y temperaturas para diferentes planos dentro del dominio computacional para tres diferentes regímenes de Reynodls. 
DOI: http://dx.doi.org/10.22201/fi.25940732e.2010.11n1.005

Análisis numérico tridimensional de la convección forzada para un flujo laminar sobre un escalón al frente

Descriptores: convección forzada, flujo laminar, escalón al frente, volúmenes finitos, SIMPLE, análisis numérico.

\begin{abstract}
A finite volume discretization technique is used to develop a numerical code to simulate the flow structures and forced convection in a forward facing step channel. The velocity field and pressure distribution inside the computacional domain are linked by the SIMPLE algorithm. The duct dimensions are defined in terms of the step height, such that the aspect and expansion ratios are four and two respectivelly. The total length in the streamflow direction is sixty times the step height, while the stepedge is located twenty times the step height after the channel inlet. The boundary conditions at the channel inlet correspond to a fully developed flow at a constant temperature $T_{0}$. The heating conditions are those of considereing the bottom wall at a high constant temperature $\left(T_{w}>T_{0}\right)$ and the other walls as adiabatic ones.

Results for the location and size of the re-circulating zones, as well as the flow structures and temperature distributions at different planes inside the computational domain for three different Reynolds parameters are presented.
\end{abstract}

Keywords: Forced convection, laminar flow, forward-facing step, finite volume, SIMPLE algorithm, numerical analysis.

\section{Introducción}

La separación del flujo y su adherencia son fenómenos que frecuentemente se encuentran presentes en aplicaciones y equipos industriales, tales como en el enfriamiento de piezas de equipo electrónico, el enfriamiento de reactores nucleares, enfriamiento de alabes de turbina, flujo en cámaras de combustión y mezclado, flujo en paredes verticales con aletas, flujo en válvulas y difusores, sólo por mencionar algunos ejemplos. En algunas otras situaciones, la separación se induce para favorecer las condiciones de transferencia de calor como puede suceder en el caso de intercambiadores de calor compactos y en algunos casos se utiliza para tener un mejor entendimiento del punto de transición de la turbulencia en convección natural y convección mixta.

En la última década con la ayuda de los equipos computacionales se han realizado una cantidad importante de estudios para tener un mejor conocimiento y entendimiento de las condiciones hidrodinámicas de la separación y adherencia del flujo. En este aspecto, es el escalón hacia atrás (backward-facing-step) el principal problema a estudiar para muchos investigadores, lo que ha llevado a este problema a considerarse como un problema tipo para la validación de códigos y procedimientos numéricos (Blackwell and Pepper, 1992; Williams and Baker, 1997). En contra parte, la geometría del escalón al frente (forward-facing-step) ha sido estudiada con mucho menor frecuencia como lo mencionan Stuer y sus colaboradores (Stuer et al., 1999) en su publicación.
Añaden que ni la topología del fenómeno, ni el tamaño y formación de las zonas de recirculación pueden predecirse con seguridad. Por otra parte, Abu Mulaweh (2003) establece en su trabajo, que el fenómeno de convección sobre el escalón hacia al frente es un tema que no ha sido estudiado debido a su complejidad. Concluye que dependiendo de la magnitud del Reynolds, es posible encontrar una o dos zonas de separación del flujo en zonas contiguas al escalón.

Algunos autores han realizado investigaciones para analizar las estructuras de flujo que se forman en un escalón al frente. Ratish y Naidu (1993) plantean una formulación de función de corriente-vorticidad para resolver las ecuaciones de Navier Stokes en dos dimensiones para flujo laminar. En su publicación no incluyen la relación geométrica del dominio computacional y por lo tanto, sus resultados son difíciles de reproducir. De manera análoga, Houde et al. (1994) utilizan una formulación de función de corriente-vorticidad y un esquema de diferencias de segundo orden para analizar numéricamente el problema de flujo laminar sobre un escalón en dos dimensiones para flujo estable. Ellos encontraron una zona de recirculación en la orilla del escalón y también encontraron que el flujo se separa de la pared inferior en un punto previo al escalón. Aún cuando sus resultados presentan una excelente aproximación con resultados previos en literatura, sus aproximaciones son para un problema en dos dimensiones y no pueden ser utilizados para validar este trabajo. Algunos otros autores han realizado estudios para la geometría del escalón 
al frente para analizar el problema de la convección natural en paredes verticales (Asseban et al., 2000) o para estudiar la convección mixta para un arreglo vertical en dos dimensiones como lo presentan Abu Mulaweh y su grupo de trabajo en diversas publicaciones (Abu-Mulaweh et al., 1996, 1994 y 1993). Aún cuando se ha realizado un importante esfuerzo por entender este fenómeno, la mayoría de las publicaciones se limitan al caso en dos dimensiones. En este trabajo, el análisis de la tridimensionalidad del problema es el principal objetivo discutido en las secciones posteriores.

La importancia de estudiar el flujo sobre un escalón al frente es por que este fenómeno se encuentra en aplicaciones industriales que tienen que ver con el aumento de la transferencia de calor y masa, en el diseño de equipos de enfriamiento en aplicaciones electrónicas, en el diseño de construcciones y edificios, en el diseño de sistemas de flujo dinámico, como lo mencionan Stuer et al. y Ravindran en sus respectivas publicaciones (1999 y 2002).

\section{Modelo matemático}

El flujo en un ducto horizontal que encierra un escalón tridimensional al frente se simuló numéricamente por medio de un código numérico en Fortran90 utilizando la técnica de los volúmenes finitos. La relación de aspecto y la relación de expansión del ducto están en función de la altura del escalón $(\mathrm{s}=0.01 \mathrm{~m})$ y tienen valores de $\mathrm{AR}=4$ y $\mathrm{ER}=2$, respectivamente.

La orilla del escalón se localiza veinte veces la altura del mismo corriente abajo de la entrada del conducto $(l=20 \mathrm{~s})$, mientras que la longitud del conducto en la dirección principal del flujo es de sesenta veces la altura del escalón $(\mathrm{L}=60 \mathrm{~s})$. Un esquema de la geometría propuesta se presenta en la figura 1.
A la entrada del ducto el flujo es considerado como un flujo tridimensional completamente desarrollado de acuerdo a la correlación presentada por Shah y London (1978). En las paredes sólidas del dominio computacional se considera la condición de frontera de no deslizamiento, mientras que las propiedades termo físicas del fluido se consideran como constantes y son evaluadas a la temperatura ambiente $T_{0}\left(T_{0}=293 \mathrm{~K}\right)$. La condición de calentamiento fue impuesta únicamente a la pared inferior del ducto, la cual se mantuvo a una temperatura constante $T_{w}\left(T_{w}=350 K\right)$, mientras que todas las demás paredes se consideraron como superficies adiabáticas. El problema se considera como uno de flujo estable, por lo que las ecuaciones que gobiernan el fenómeno se reducen a las siguientes formas (Kakac and Yener, 1995).

Conservación de la masa:

$\rho \nabla \cdot \vec{V}=0$

Ecuación de momento

$$
\rho V \cdot(\nabla \vec{V})=-\nabla p+\mu \nabla \cdot(\nabla \vec{V})
$$

Ecuación de la energía

$$
V \cdot \nabla(\rho C p T)=\nabla \cdot(k \nabla T)
$$

\section{Procedimiento numérico}

Para la elaboración del código numérico, las ecuaciones de momento y de la energía, fueron discretizadas por medio de la técnica de los volúmenes finitos. El campo de velocidades y la distribución de presión fueron ligadas por medio del algoritmo SIMPLE (Patankar, 1980), mientras que el esquema de la ley de potencias (Patankar,

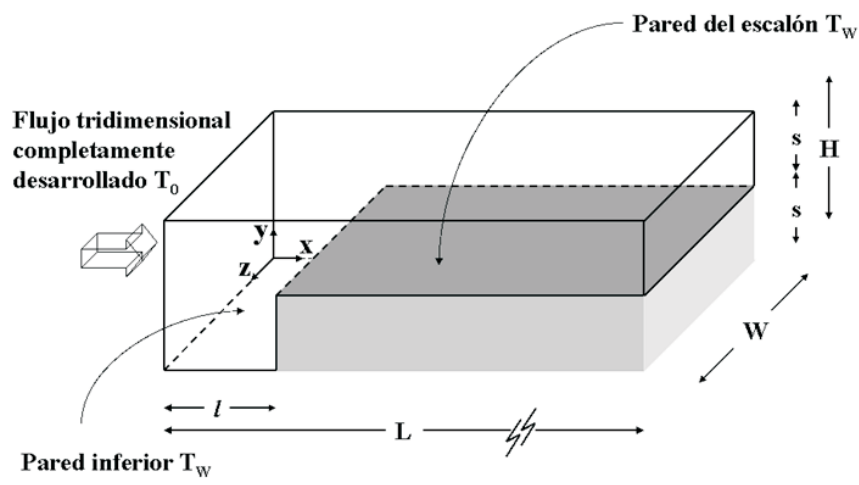

Figura 1. Dominio computacional para el ducto tridimensional con escalón al frente 
DOI: http://dx.doi.org/10.22201/fi.25940732e.2010.11n1.005

Análisis numérico tridimensional de la convección forzada para un flujo laminar sobre un escalón al frente

1980) se empleó para representar los términos de convección-difusión en la interfase de cada volumen de control. Para resolver el problema se empleó una malla dislocada, en la cual los nodos principales se destinaron para almacenar las propiedades termofísicas, la temperatura y la presión, mientras que en la interfase de cada nodo se almacenó la variable para representar la velocidad en cada dirección coordenada. A la salida del ducto se impusieron las condiciones naturales de flujo desarrollado para cada variable.

El bloque sólido (escalón) dentro del dominio computacional se simuló escogiendo un valor muy grande para el coeficiente de difusión de la ecuación de momento $\left(\mu=10^{50}\right)$, mientras que para la ecuación de la energía se consideró el valor de conductividad térmica del cobre $\left(\mathrm{k}=386 \mathrm{Wm}^{-1} \mathrm{~K}^{-1}\right)$. Para la interfase sólido-fluido los coeficientes de difusión se evaluaron por medio de una media armónica de las propiedades en los volúmenes de control adyacentes según describe Patankar (1980).

El algoritmo de Thomas (TDMA) y una combinación de barridos línea por línea y plano por plano se implementó en cada coordenada para calcular la velocidad y presión dentro del dominio computacional. Factores de relajamiento fueron impuestos para los componentes de velocidad $\left(\alpha_{u}=\alpha_{v}=\alpha_{w}=0.4\right)$ y para la presión $\left(\alpha_{p}=0.4\right)$ para asegurar convergencia en el proceso numérico. Durante el proceso numérico se vigilaron los residuos normalizados para los componentes de velocidad y presión, y se declaró solución del problema una vez que estos reportaron valores del orden de $1 \times 10^{-8}$ (Barbosa et al., 2005a).

Se consideró una malla no uniforme para resolver el problema. En este sentido, en la vecindad de las paredes sólidas se colocaron los volúmenes de control más pequeños y conforme se sitúa en una posición más alejada de las paredes sólidas el tamaño se va incrementando. El tamaño de los volúmenes de control crece en función del tamaño de volumen de control que le precede. Una descripción más detallada de esta implementación se puede encontrar en algunos trabajos previamente publicados por los autores (Barbosa et al., 2005a y 2005b).

El estudio de independencia de malla correspondiente, se llevó acabo para diferentes densidades de volúmenes de control utilizando las condiciones de cómputo más severas $(R e=800)$ y el punto en el plano central en la dirección transversal del ducto $(z / W=0.5)$, donde la componente en la dirección x para el esfuerzo cortante en la pared inferior era igual a cero $(\tau=0)$ se tomó como el parámetro para monitorear y declarar independencia de malla. Los estudios indicaron que una malla de 150:40:40 (x:y:z) tiene diferencias porcentuales relativas del orden del $0.5 \%$ cuando se compara con una malla de 180:40:40 (x:y:z). Por lo tanto, la primera de ellas fue propuesta como malla para las corridas de producción de resultados numéricos. Es importante mencionar que el incremento de nodos en la dirección vertical (coordenada y) y transversal (coordenada z) no presenta variaciones considerables en el estudio de independencia de malla (Barbosa et al., 2007).

Una vez establecido el procedimiento para el estudio de independencia de malla, el siguiente paso fue el de encontrar un procedimiento para validar el código numérico. Una validación directa del código no pudo llevarse a cabo, debido a la falta de información concerniente a este problema en la literatura especializada. Sin embargo, se observó que la única diferencia en la implementación numérica entre un problema con el escalón hacia atrás y un escalón al frente es la posición del mismo en el ducto. La primera de ellas se refiere a un escalón a la entrada del ducto, mientras que la segunda de ellas se refiere a un escalón a la salida del mismo. Por lo tanto, si el código y la técnica numérica son validados para el caso del escalón hacia atrás, entonces el mismo código con sus respectivos cambios puede ser utilizado para resolver el problema del escalón al frente. En este sentido, el problema de convección forzada para un ducto tridimensional con un escalón hacia atrás fue estudiado y simulado numéricamente con la misma técnica y código numérico y los resultados fueron presentados previamente por los autores (Barbosa, 2005b), en los cuales se reportaron errores menores al $2 \%$ cuando se comparó con los resultados experimentales publicados por Armaly y su equipo de trabajo (Armaly et al., 2003). Por lo tanto, se puede concluir que el código y la técnica numérica utilizada es altamente precisa y de confiabilidad para estudiar el caso de flujo sobre un escalón hacia atrás y su extensión al caso de estudio de un escalón hacia al frente. Mayor información del proceso de validación puede encontrarse el trabajos presentados por los autores (Barbosa et al., 2005a, 2005b y 2007).

\section{Análisis de resultados numéricos y discusión}

El estudio numérico en el presente trabajo considera el estudio de flujo a través de un ducto tridimensional que encierra un escalón hacia al frente para tres diferentes parámetros de Reynolds ( $R e=200,400$ y 800). El origen coordenado para la geometría en cuestión se sitúa de acuerdo a la figura 1.

Un concepto utilizado ampliamente para la caracterización de la separación del flujo es la delimitación de la zona de recirculación primaria o la zona en la cual la 
componente en la dirección principal del flujo del esfuerzo cortante sobre la pared es negativa. La razón por la cual se dan estos valores es porque dentro de la zona de recirculación la componente principal de velocidad del flujo toma valores negativos. Para el caso del escalón hacia al frente una zona de recirculación se desarrolla sobre la pared inferior del ducto en la zona previa al escalón $(x<0.2)$. Los resultados numéricos de la distribución de estas zonas para $\operatorname{Re}=200,400$ y 800 se presentan en las figuras $2 \mathrm{a}$ ), 2b) y $2 \mathrm{c}$ ), respectivamente.

a)

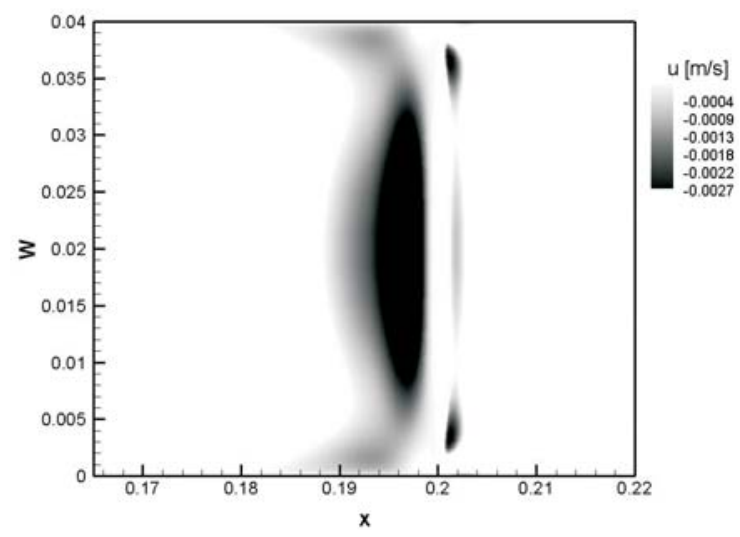

b)

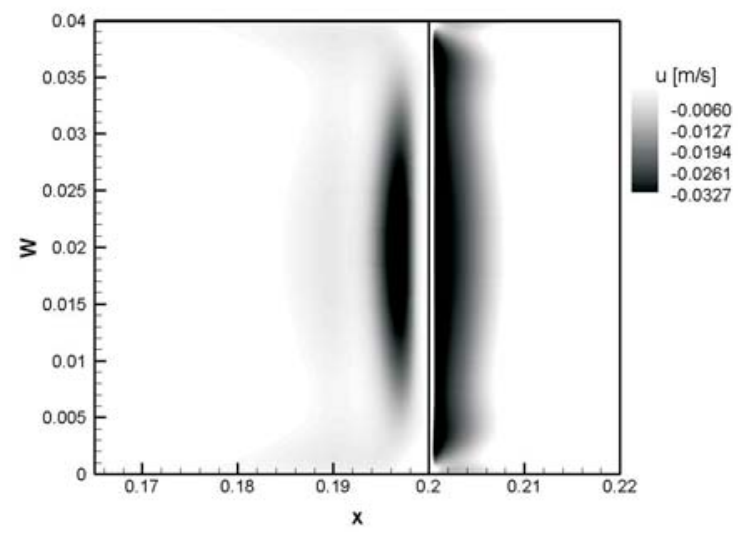

c)

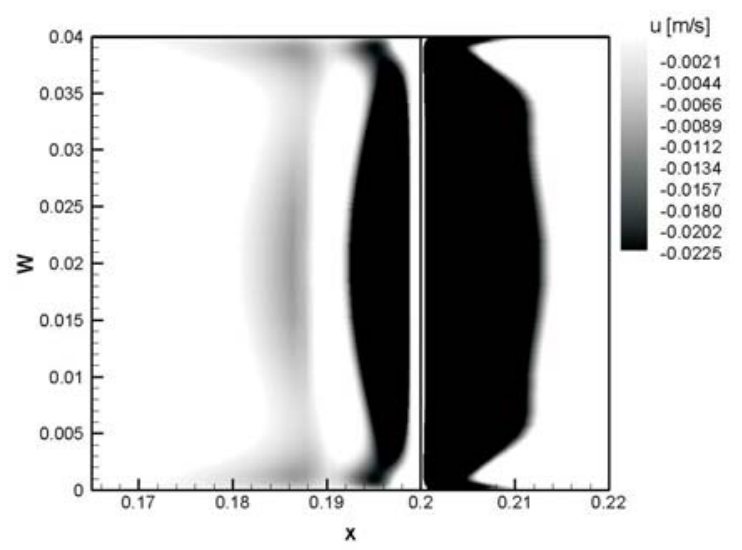

Figura 2. Zonas de re-circulación en un plano horizontal adyacente a la pared inferior y a la pared del escalón a) $R e=200$, b) $R e=400$ y c) $R e=800$ 
DOI: http://dx.doi.org/10.22201/fi.25940732e.2010.11n1.005

Análisis numérico tridimensional de la convección forzada para un flujo laminar sobre un escalón al frente

En estas figuras las zonas sombreadas indican que la componente de velocidad en la dirección de flujo principal tiene valores negativos. La zona de recirculación corriente arriba de la orilla del escalón $(x<2.0)$ presenta un comportamiento simétrico respecto al eje central $(\mathrm{z} / \mathrm{W}=0.5)$ en la dirección transversal del ducto. La separación de flujo ocurre más próxima a la entrada del ducto si el número de Reynolds es más elevado. En esta figura también se muestra que cerca de las paredes laterales se encuentran los valores de las posiciones $(\mathrm{x} / \mathrm{s})$ de separación de flujo más cercanas a la entrada del ducto. Este efecto se puede asociar a la condición de no-deslizamiento impuesta en la simulación numérica. Para $R e=800$ y $R e=400$, se puede apreciar una zona muy pequeña de valores positivos para la componente de velocidad $u$ antes del escalón ( $x \cong 0.19)$. Este particular fenómeno no se observa para $\mathrm{Re}=200$. Por lo tanto, se puede decir que para $\operatorname{Re}=800$ y $\operatorname{Re}=400$ la zona de re-circulación previa al escalón no termina en la orilla del mismo $(x / s=20)$, sino un punto previo.

White (1999) establece que el flujo que pasa un obstáculo se separa, y en algún lugar corriente abajo va a reunirse, formándose por lo tanto, una zona de recirculación en la cual la componente de velocidad en la dirección de flujo principal toma valores negativos. Este fenómeno fue detectado por la simulación numérica y los resultados de las zonas de recirculación que se forman sobre la pared del escalón $(x>0.20)$ para los diferentes parámetros de Reynolds (figuras $2 \mathrm{a}$ ), 2b), y 2c). Como se evidencia en la figura 2, el tamaño de esta zona en la dirección coordenada $\mathrm{x}$ es del orden de unos cuantos centímetros y la tendencia de ésta es similar para los diferentes casos en estudio. De acuerdo a la figura 2, las zonas de recirculación de mayor tamaño corresponden a $R e=800$, mientras que las más pequeñas corresponden a $R e=200$. También se observa de esta figura que la zona de separación sobre el escalón es casi una línea recta. La razón de este comportamiento se asocia con el hecho de que el cambio abrupto en la dirección transversal del ducto está siempre a la misma posición (orilla del escalón), es decir, es una línea recta. Sin embargo, la línea que delimita el final de la zona de recirculación sobre el escalón en la dirección transversal del ducto no es una línea recta. Este hecho se asocia con la formación de zonas altamente tridimensionales que se generan en esta zona. Como se observa en la figura 2c) para $\mathrm{Re}=800$, la línea que delimita la zona de recirculación sobre el escalón, presenta más irregularidades en la dirección z que el resto de los casos, debido a los efectos altamente tridimensionales para este régimen de flujo.
Para dar más detalle de las estructuras de flujo en el fenómeno a estudiar, en las figuras 3, 4 y 5 se muestran las líneas de corriente y las zonas en la cuales la componente principal del flujo presenta valores negativos para $R e=200,400$ y 800, respectivamente. Las figuras antes mencionadas presentan claramente el salto hidráulico del fluido en la orilla del escalón. El fenómeno se hace más evidente cuando el Reynolds se incrementa. Posterior a este punto las líneas de corriente muestran que el flujo continúa su desarrollo hacia la salida del conducto. Especial atención requiere la figura 5 para $R e=800$. En esta figura se observa que algunas líneas de corriente saltan sobre el escalón, pero su momento es tan grande que impactan la pared superior del ducto y posteriormente se mueven hacia la pared horizontal del escalón para reunirse con el flujo y desplazarse a la salida del conducto en la zona horizontal adyacente a la pared del escalón. Si se compara con el comportamiento para $R e=400$ y $R e=200$, se observa que las líneas de corriente saltan el escalón y permanecen adyacentes a la pared superior y se conservan en esta zona hasta la salida del conducto. La diferencia entre tales comportamientos se asocia al mayor momento que presentan el flujo con mayor Reynolds.

Asimismo, las gráficas en las figuras 3, 4 y 5 muestran las zonas de recirculación dentro del dominio computacional para los casos en estudio. Se observa que a mayor número de Reynolds, las zonas de recirculación presentan mayor extensión, tanto en la dirección vertical como en la dirección horizontal.

Los mapas de temperatura dentro del dominio computacional obtenidos durante la simulación numérica se muestran en las figuras 6, 7 y 8 para $R e=200,400$ y 800 , respectivamente. Se muestran distribuciones de temperatura en planos horizontales, uno adyacente a la pared inferior del ducto $(\mathrm{y} / \mathrm{s}=0.017)$ y el otro adyacente al plano del escalón (y/s=1.07). Se observa que en el caso del plano $\mathrm{y} / \mathrm{s}=1.07$ existen regiones de temperaturas elevadas en la vecindad de las paredes laterales $(z=0.0 \mathrm{y}$ $z=0.40$ ). Estás zonas se hacen más evidentes si el número de Reynolds aumenta. Por otra parte, la distribución de temperaturas en el plano $\mathrm{y} / \mathrm{s}=0.17$ para el caso de $\mathrm{Re}=200$ (figura 6) muestra cómo el flujo va incrementando su temperatura a medida que se avanza en la dirección $\mathrm{x}$.

En esta figura se observa que la zona de iso-temperaturas adyacente a las paredes laterales se extiende hacia la entrada del ducto. Este fenómeno es similar al comentado en la figura 2a) para la zona de recirculación previa al escalón. La razón se atribuye a que en la zona de recirculación se encuentran mayores relaciones de 
transferencia de calor y por lo tanto, deben existir zonas de más altas temperaturas.

La tendencia de la distribución de temperaturas para el plano adyacente a la pared inferior es similar para $\mathrm{Re}=400$ y $\mathrm{Re}=800$ a la comentada para $\mathrm{Re}=200$ en el párrafo anterior. Sin embargo, para $\mathrm{Re}=800$ (figura 8) se observa que entre la orilla del escalón $(x=0.2)$ y una franja de temperatura alta (zona amarilla $T \cong 335 \mathrm{~K}$ ) hay una zona de temperatura media (color verde $\mathrm{T} \cong 320 \mathrm{~K}$ ). Este particular fenómeno se asocia con la zona de valores positivos para la componente principal de flujo presente en la zona de recirculación previa al escalón y que se indicó en la figura $2 \mathrm{c}$ ).

Es importante notar que esta circunstancia para la distribución de temperaturas también se encuentra presente aunque de una forma menos evidente para $R e=400$ y su causa se asocia de igual forma a la franja de valores de velocidad positiva para la componente de flujo principal dentro de la zona de recirculación mostrados en la figura $2 b$ ).

Finalmente, en la figura 9 se muestra la distribución del número de Nusselt promediado sobre la dirección transversal para diferentes posiciones en la dirección axial. Se observa que la tendencia de la distribución es muy similar para cada caso estudiado, teniéndose los valores más elevados para los mayores números de Reynolds. A la entrada del conducto, la distribución se comporta como una distribución clásica de problema de flujo con transferencia de calor en un conducto.

Sin embargo, en la vecindad del escalón la distribución disminuye teniéndose valores cercanos al valor de cero. Este particular comportamiento se asocia con la zona de recirculación previa al escalón.

Posteriormente, se observa que en la distribución el valor del número de Nusselt se incrementa exponencialmente hacia su valor máximo, debido a la separación de flujo causada por el cambio abrupto en la geometría. Una vez que el número de Nusselt alcanza su valor máximo, entonces decrece asimptóticamente hacia su valor de flujo completamente desarrollado a la salida del conducto.

Se observa en la gráfica que para $\mathrm{Re}=200$ el conducto es lo suficientemente largo para acomodar flujo térmico completamente desarrollado a la salida, no siendo el caso para $R e=400$ y 800 . De acuerdo a los resultados presentados a lo largo de este trabajo, para $R e=400$ y 800 existe una zona de recirculación posterior a la orilla del escalón y adyacente a la pared horizontal del mismo. Por lo tanto, el desarrollo de la capa límite hidráulica y térmica no empieza a la orilla del escalón para estos dos regímenes de Reynolds, sino a una posición corriente abajo, causando que el conducto no sea lo suficientemente largo para acomodar flujo térmico completamente desarrollado.

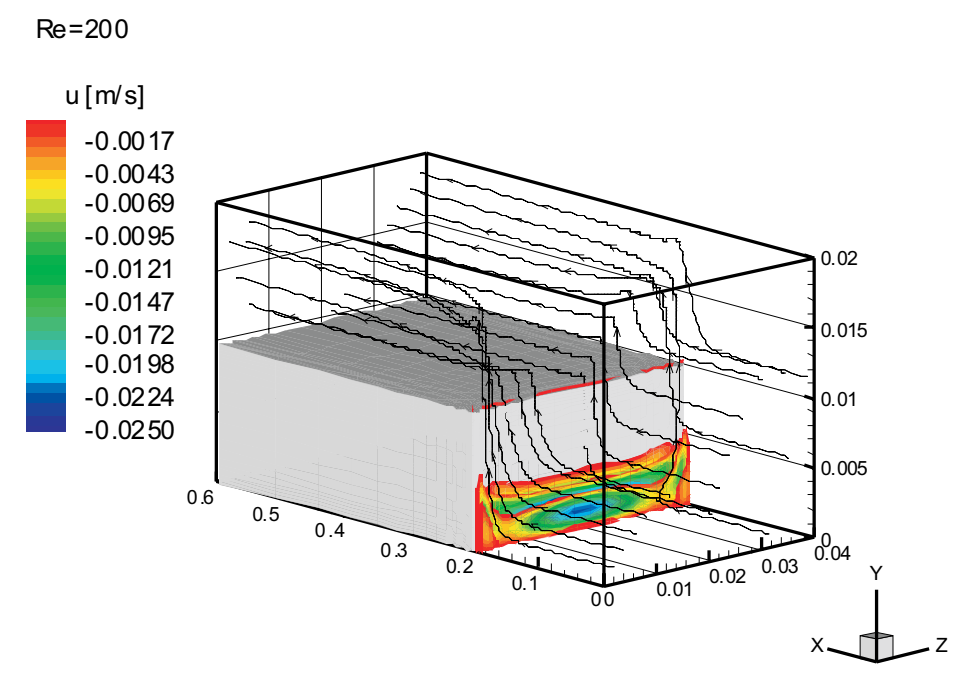

Figura 3. Lineas de corriente y zonas de recirculación para $R e=200$ 


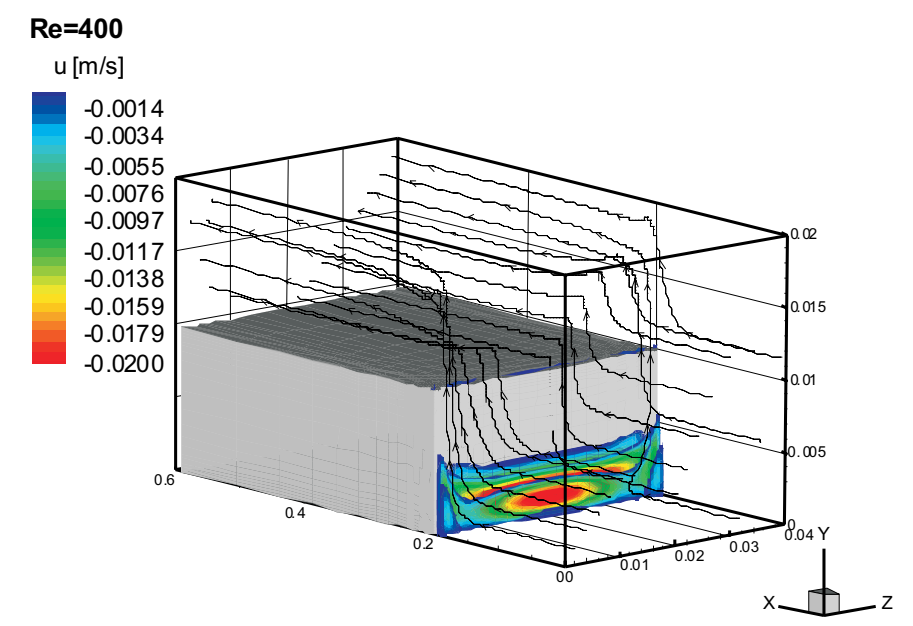

Figura 4. Lineas de corriente y zonas de recirculación para $R e=400$ $\operatorname{Re}=800$

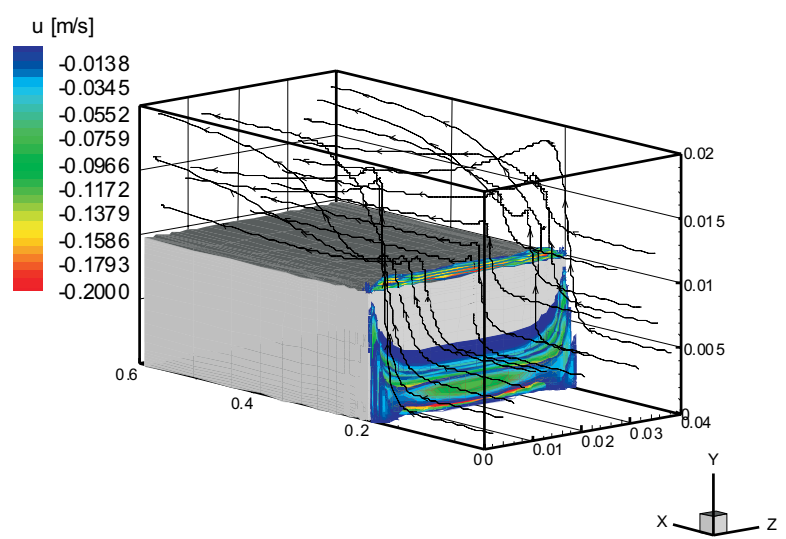

Figura 5. Lineas de corriente y zonas de recirculación para $R e=800$

a)

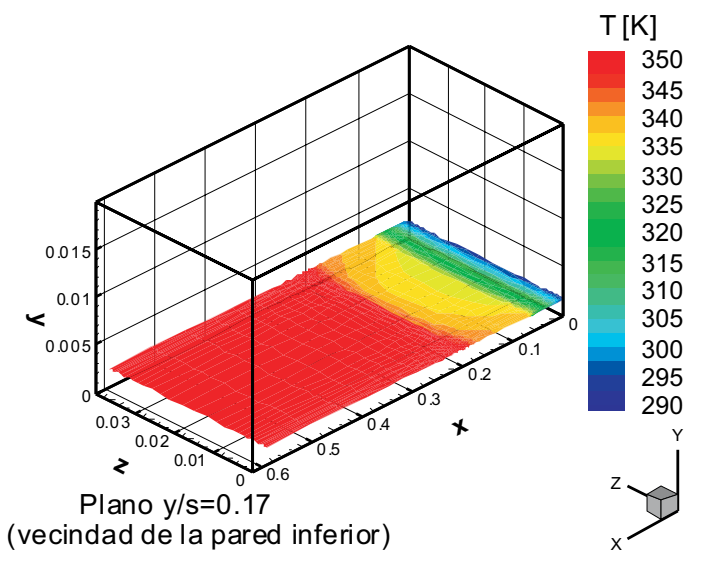

b)

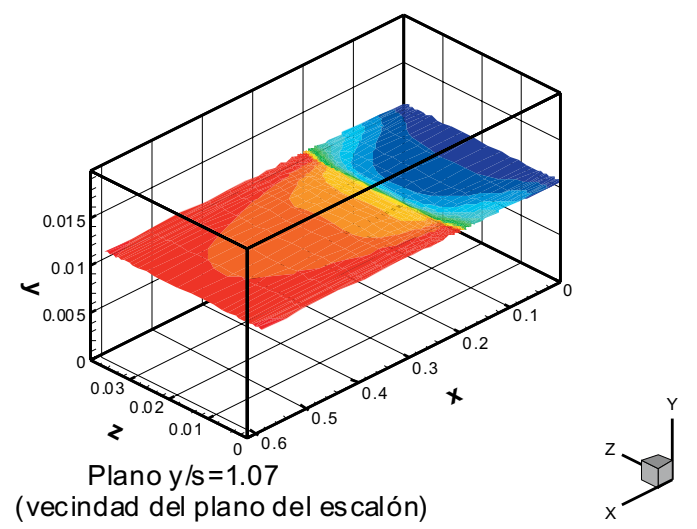

Figura 6. Distribución de temperaturas para $\operatorname{Re}=200$ a) plano $\mathrm{y} / \mathrm{s}=0.17, \mathrm{~b}$ ) plano $\mathrm{y} / \mathrm{s}=1.07$ 
a)

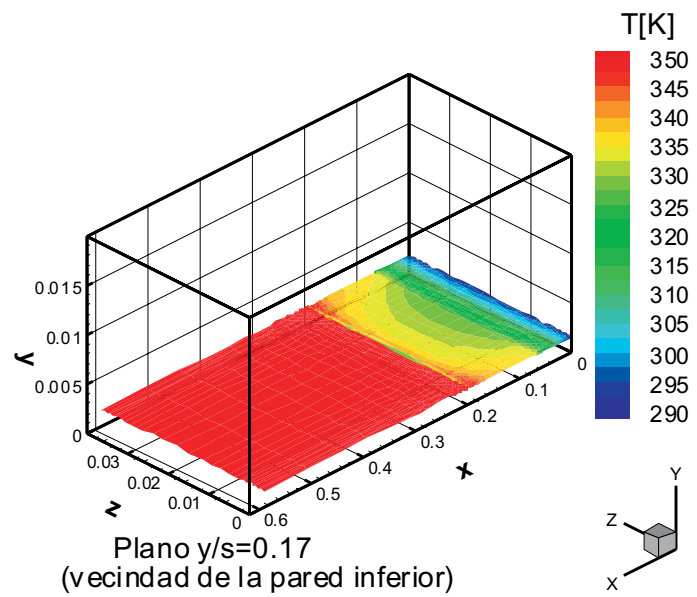

b)

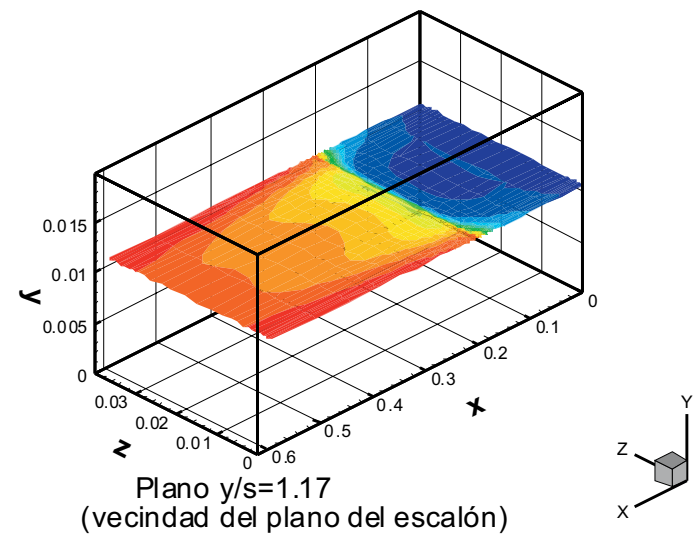

Figura 7. Distribución de temperaturas para $R e=400$ a) plano $\mathrm{y} / \mathrm{s}=0.17, \mathrm{~b}$ ) plano $\mathrm{y} / \mathrm{s}=1.07$

a)

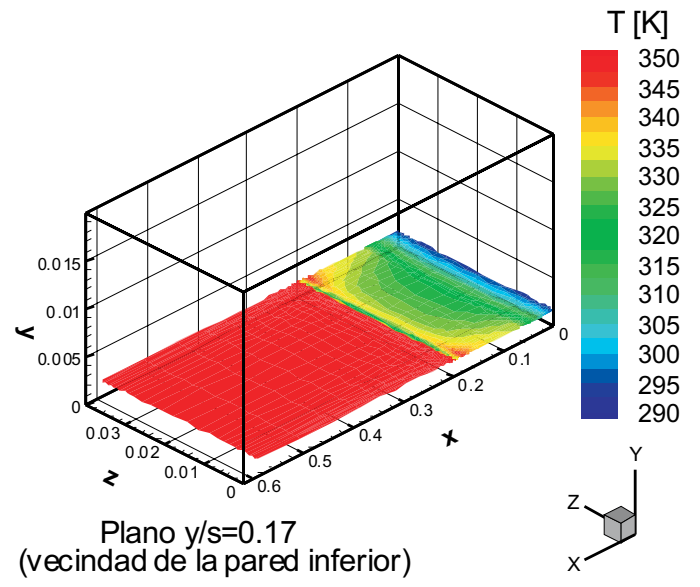

b)

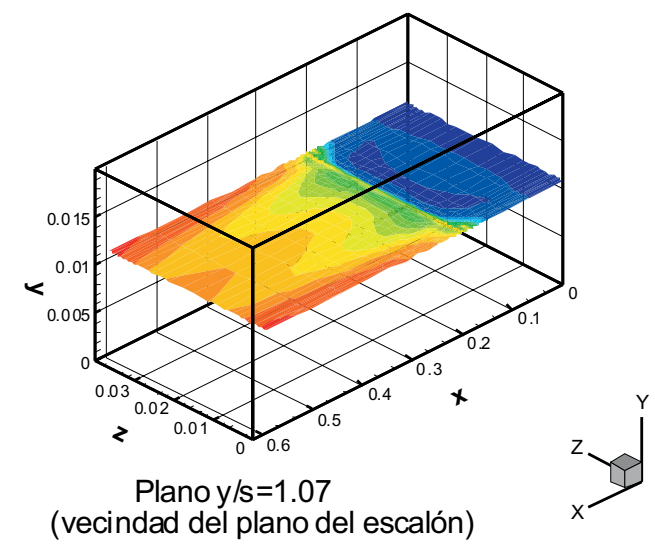

Figura 8. Distribución de temperaturas para $R e=800$ a) plano $\mathrm{y} / \mathrm{s}=0.17$, b) plano $\mathrm{y} / \mathrm{s}=1.07$

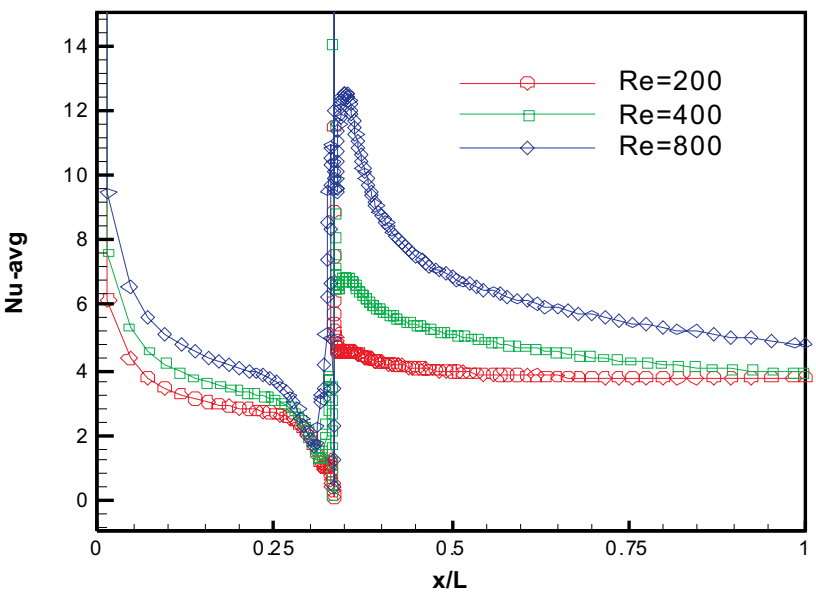

Figura 9. Nusselt promedio en la dirección transversal 
DOI: http://dx.doi.org/10.22201/fi.25940732e.2010.11n1.005

Análisis numérico tridimensional de la convección forzada para un flujo laminar sobre un escalón al frente

\section{Nomenclatura}

AR relación de aspecto, $\mathrm{W} / \mathrm{s}$

ER relación de expansión, $\mathrm{H} / \mathrm{s}$

$\mathrm{H}$ altura del conducto, 2s [m]

$1 \quad$ longitud de entrada del conducto, 20s [m]

L longitud del conducto en la dirección principal de flujo, 60s $[\mathrm{m}]$

$N u_{-a v g} N u=(d \bar{T} / d y)_{y=0} 2 H /\left(T_{w}-T_{b}\right)$

p presión $[\mathrm{Pa}]$

Re número de Reynolds $\operatorname{Re}=2 \rho U_{0} s / \mu$

$\mathrm{s} \quad$ altura del escalón, [0.01m]

$\mathrm{T}_{0} \quad$ temperatura ambiente $[293 \mathrm{~K}]$

$\mathrm{V} \quad$ velocidad $[\mathrm{m} / \mathrm{s}]$

W ancho del conducto, $4 \mathrm{~s}[\mathrm{~m}]$

x dirección principal del flujo dirección/coordenada

y dirección transversal del flujo dirección/coordenada

z dirección transversal del flujo dirección/coordenada

letras griegass

$\mu \quad$ viscosidad dinámica del flujo

$\left[1.81 \times 10^{-5} \mathrm{kgm}^{-1} \mathrm{~s}^{-1}\right]$

$\rho \quad$ densidad del flujo $\left[1.205 \mathrm{kgm}^{-3}\right]$

Subíndices

b bulk

0 condiciones de entrada

W condiciones en la pared o de frontera

\section{Conclusiones}

Se presentaron algunos resultados de la simulación numérica de flujo de aire dentro de un conducto horizontal que encierra un escalón al frente para tres parámetros de Reynods ( $R e=200,400$ y 800) y las mismas condiciones de calentamiento. Las estructuras de flujo indican que el flujo se separa en dos zonas diferentes del dominio computacional dando lugar a la formación de dos zonas de recirculación. Una zona de recirculación se encuentra en la región adyacente a la pared inferior del ducto y previa al escalón, mientras que la otra, se forma corriente abajo de la orilla del escalón sobre la pared horizontal del mismo. El tamaño y localización de estas zonas es dependiente del número de Reynods a considerar. A medida que el número de Reynolds crece, las zonas de recirculación crecen en tamaño. También se observa que a medida que Reynolds es incrementado, las estructuras de flujo se vuelven más complejas y altamente tridimensionales.

\section{Referencias}

Abu-Mulaweh H.I. A Review of Research on Laminar Mixed Convection Flow Over a Backward and Forward-Facing Steps. International Journal of Thermal Sciences, 42:897909.2003.

Abu-Mulaweh H.I., Armaly B.F., Chen T.S. Measurements in Buoyancy-Opposing Laminar Flow Over a Vertical Forward-Facing Step. International Journal of Heat and Mass Transfer, 39:1805-1813. 1996.

Abu-Mulaweh H.I., Armaly B.F., Chen T.S., Hong B. Mixed Convection Adjacent to a Vertical Forward-Facing Step. Proceedings of the 10th International Heat Transfer Conference, 5, pp.423-428, 1994.

Abu-Mulaweh H.I., Armaly B.F., Chen T.S. Measurements of Laminar Mixed Convection Flow Over a Horizontal Forward-Facing Step. AIAA Journal of Thermophysics and Heat Transfer, 7:569-573. 1993.

Armaly B.F., Li A., Nie J.H. Measurements in Three-Dimensional Laminar Separated Flow. International Journal of Heat and Mass Transfer, 46:3573-3582. 2003.

Asseban A., Lallemand M., Saulnier J.B., Fomin N., Lavinskaja E., Merzkirch W., Vitkin D. Digital Speckle Photography and Speckle Tomography in Heat Transfer Studies. Optics and Laser Technology, 32:583-592. 2000.

Barbosa-Saldana J.G., Anand N.K., Sarin V. Numerical Simulation of Mixed Convective Flow Over a Three-Dimensional Horizontal Backward Facing Step. ASME Journal of Heat Transfer, 127:1027-1036. 2005a.

Barbosa-Saldana J.G., Anand N.K., Sarin V. Numerical Simulation of Forced Convection Over a Three-Dimensional Horizontal Backward-Facing Step. International Journal of Computational Methods in Engineering Science and Mechanics, 6:225-234. 2005b.

Barbosa-Saldaña J.G., Quinto Diez P., Sánchez Silva F., Carvajal-Mariscal I. Numerical Simulation for the Flow Structures Following a Three-Dimensional Horizontal Forward-Facing Step Channel. Revista Mexicana de Física, 53(2):87-95. 2007.

Blackwell B.F., Pepper D.W. Benchmark Problems for Heat Transfer Codes. ASME-HTD-222: Anaheim. 1992.

Houde H., Lu J., Bao W. A Discrete Artificial Boundary Condition for Steady Incompressible Viscous Flows in a No-Slip Channel Using a Fast Iterative Method. Journal of Computational Physics, 114:201-208. 1994.

Kakac S., Yener Y. Convective Heat Transfer. Boca Raton. CRC Press, Inc. 1995. 
DOI: http://dx.doi.org/10.22201/fi.25940732e.2010.11n1.005

J.G. Barbosa-Saldaña, J.A. Jiménez-Bernal, C. del C. Gutiérrez-Torres y P. Quinto-Diez

Patankar S.V. Numerical Heat Transfer and Fluid Flow. Philadelphia. Taylor and Francis. 1980.

Ratish-Kumar B.V., Naidu K.B. A Streamline Upwinding Streamfunction-Vorticity Element Analysis of Navier-Stokes Equations. Applied Numerical Mathematics, 13:335-344. 1993.

Ravindran S.S. Control of Flow Separation Over a Forward-Facing Step by Model Reduction. Computer Methods in Applied Mechanics and Engineering, 191:4599-4617. 2002.
Shah R.K., London A.L. Laminar Flow Forced Convection in Ducts. New York. Academic Press.1978.

Stuer H., Gyr A., Kinzelbach W. Laminar Separation on a Forward Facing Step. European Journal of Mechanics B/Fluids, 18:675-692. 1999.

White F.M. Mecánica de Fluidos. Ciudad de Mexico. McGraw Hill. 1999.

Williams P.T., Baker A.J. Numerical Simulations of Laminar Flow Over a 3D Backward-Facing Step. International Journal of Numerical Methods in Fluids, 24:1159-1183. 1997.

\section{Semblanza de los autores}

Juan Gabriel Barbosa-Saldaña. Doctor en ciencias en ingeniería mecánica de la Universidad de Texas A\&M (2005). Actualmente es profesor titular de la SEPI-ESIME, Unidad Zacatenco. Dentro de su producción académica cuenta con siete publicaciones en journals del JCR data base, además de 10 publicaciones en revistas internacionales, incluyendo seis publicaciones en revistas reconocidas por el padrón de excelencia del CONACYT. Actualmente las áreas de interés incluyen la simulación numérica de flujo de fluidos y transferencia de calor, técnicas aplicadas al Open-MP, uso y aplicaciones de energías renovables.

José Alfredo Jiménez-Bernal. Es ingeniero electromecánico del Instituto Tecnológico de Acapulco (1997). Maestro en ciencias en ingeniería mecánica de la Sección de Estudios de Posgrado e Investigación de la ESIME-IPN (2000) y doctor en ciencias en ingeniería mecánica de la Universidad de Texas A\&M (2004). Actualmente es profesor titular de la SEPI-ESIME, Unidad Zacatenco. Sus áreas de interés son turbulencia y termodinámica endorreversible.

Claudia del C. Gutiérrez-Torres. Es ingeniera electromecánica del Instituto Tecnológico de Acapulco (1997). Maestra en ciencias en ingeniería mecánica de la Sección de Estudios de Posgrado e Investigación de la ESIME-IPN (2000) y doctora en ciencias en ingeniería mecánica de la Universidad de Texas A\&M (2004). Actualmente es profesora titular de la SEPI-ESIME, Unidad Zacatenco. Sus áreas de interés son termofluidos, turbulencia y sistemas de separación.

Pedro Quinto-Diez. Es ingeniero mecánico con especialidad en ingeniería mecánica en la Escuela Superior de Ingeniería Mecánica y Eléctrica del Instituto Politécnico Nacional, México. Obtuvo el diploma de estudios avanzados en Ingeniería Mecánica por la Universidad Claude Bernard de Lyon, Francia. Es doctor en ingeniería mecánica por el Instituto Nacional de Ciencias Aplicadas (INSA) de Lyon, Francia. Es profesor titular del programa de Posgrado en Ingeniería Mecánica en la especialidad de Energética en la Sección de Estudios de Posgrado e Investigación de la ESIME del Instituto Politécnico Nacional. Su experiencia profesional es en el área de aire acondicionado e ingeniería térmica. 